\title{
Optimal Image Fusion
}

\section{Shristy Khandelwal Department of \\ Electronics Engineering YIT Jaipur}

\author{
Mahendra Kumar \\ Department of \\ Electronics Engineering, \\ UCE RTU, Kota
}

\author{
Anila Dhingra \\ Department of \\ Electronics Engineering, \\ YIT Jaipur
}

\begin{abstract}
In this paper a saras-type image-fusion technique using hybrid SPIHT and SOMA. This method shows fusion of images and can be used for fusion of multi model image. It is concluded that fusion with advanced single levels offers better fusion quality. This method provides a comparative study between proposed \& literature techniques and validation of the projected algorithm as Peak Signal to Noise Ratio (PSNR), Root Mean Square Error (RMSE).
\end{abstract}

\section{Keywords}

Saras type image, Set Partitioning in Hierarchical Trees (SPIHT), PSNR, RMSE, SOMA.

\section{INTRODUCTION}

Multi sensor data fusion plays a imperative role in defence in addition to civillian applications as it involves diversity of sensors offered and those working in diverse spectral bands. A further extensive tool in signal and image processing is multi-scale disintegration. In Biomedical Science it is required to increase the information content of an image and multiple registered images are combined together to get image fusion but it is an ongoing research part. Various image fusion algorithms such as multi-resolution [1, 2], multi scale [3] and statistical signal processing $[4,5,6]$ based methods are presented and estimated. The progress in the domain of sensing technologies multi-sensor techniques have become a realism in a various fields such as machine vision, medical imaging, remote sensing and the military applications for which they were elaborated.

Image fusion presents an effectual means of reducing the growing amount of information even at the similar time extracting every valuable information from the source images. These techniques offers an increase in total amount of data accessible. Apart from sinking the quality of data, image fusion aims to craft new images so as to get more apposite for the function of human/machine perception, and for additional image- processing tasks like medical imaging and remote sensing also in applications like segmentation, target detection or object exposure. Multi-sensor data frequently offers corresponding information, so image fusion imparts an effectual method for analysis of data and to enable comparison. For eg. Visible-band and infrared pictures may be fused to aid pilots landing aircraft in deprived visibility [3, 5,7]. At the end, the functioning of the image fusion schemes are predicted as tradeoffs between true image \& fused image. In preceding techniques when apply fuzzy type images, the performance standard is deprived. As a result this paper proposed a novel Curvelet transform techniques which provide fused image with superior quality. The paper remainder is prearranged as follows: Section II illustrates SPIHT and SOMA image compression technique. Section III describes different Fusion Performance evaluation criterion . Section IV describes Results and comparatively study of techniques. Section V present concluding remarks.

\section{PROPOSED SPIHT AND SOMA TECHNIQUE}

The important advantages of SPIHT method are it can provided superior Image quality with larger PSNR and smaller RMSE[11].

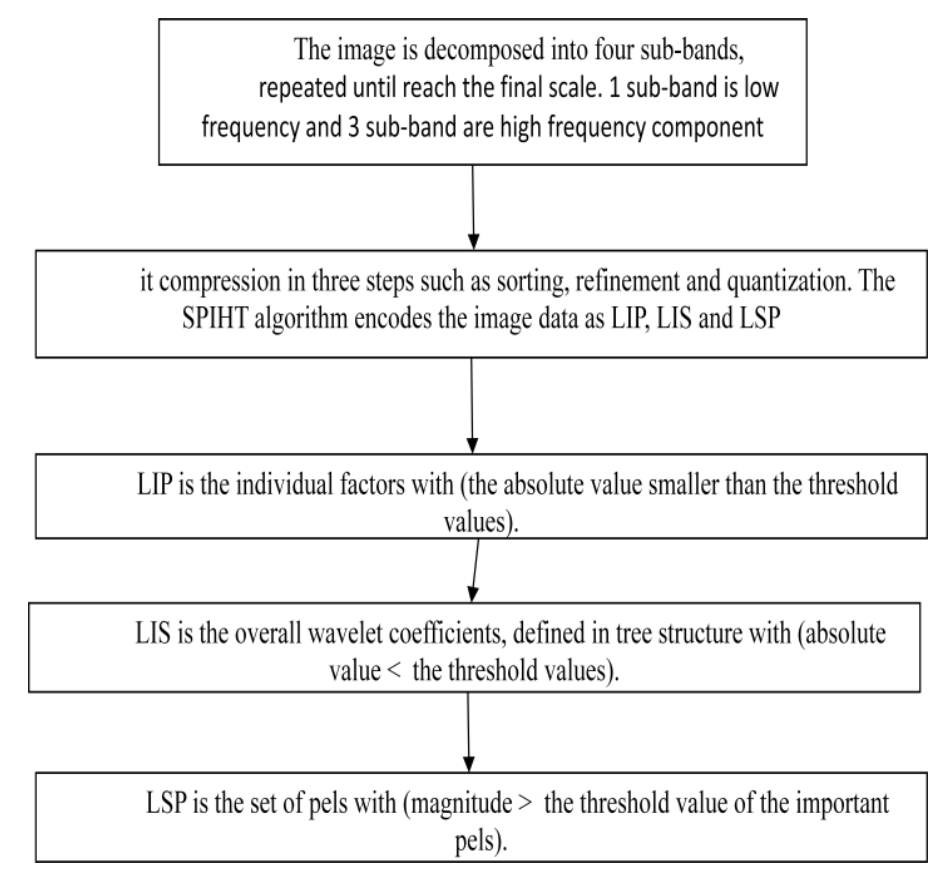

Fig1. SPIHT Algorithm

\section{PROPOSED IMAGE FUSION SYSTEM}

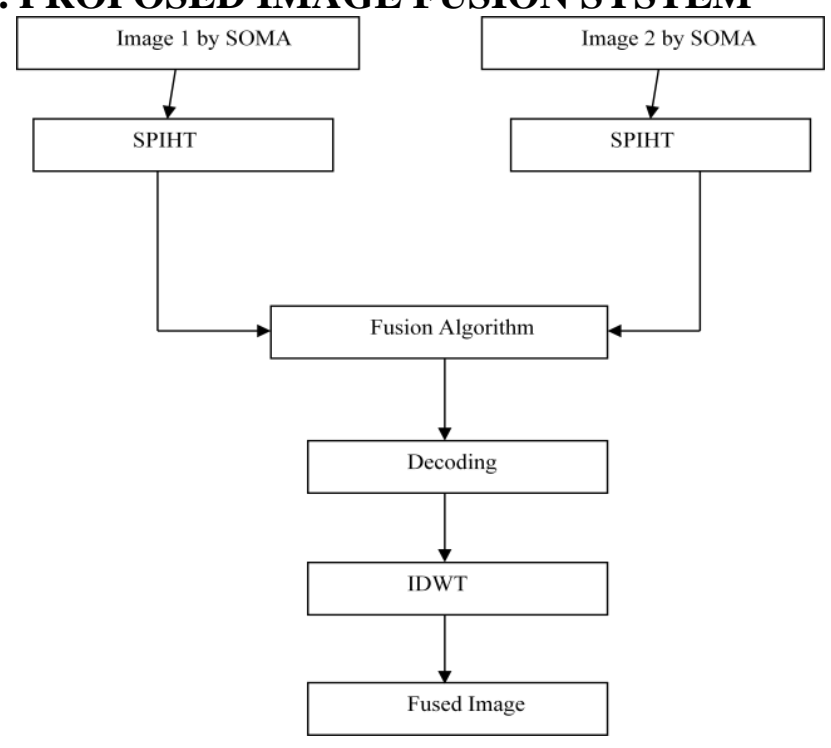

Fig.2 (a): Proposed Image Fusion technique 
Consider image 1 as fuzzy type image 1 and image 2 as fuzzy type image 2 and image compression and feature extracted by SPIHT technique and apply Image fusion algorithm as averaging method for fused image and compare actual/true image with fused image and calculate PSNR and RMSE to check effectiveness of proposed system [15-20].

Pseudo code:

Im1=DWT (image 1$)$

En1=encoding $(\operatorname{Im} 1)$

De1=decoding $(\operatorname{En} 1)$

$\operatorname{Im} 1 '=\operatorname{IDWT}(\mathrm{De} 1)$

Id1=Im1-Im1'

$\operatorname{Im} 2=$ DWT (image2)

En2=encoding $(\operatorname{Im} 2)$

De2=decoding $($ En2)

$\operatorname{Im} 2 '=\operatorname{IDWT}($ De2)

$\operatorname{Id} 2=\operatorname{Im} 2-\operatorname{Im} 2{ }^{\prime}$

$\mathrm{Id}=\operatorname{abs}(\mathrm{Id} 1)-(\mathrm{Idf})>=0$

$\operatorname{Imf}=\mathrm{Idf}+\operatorname{Imf}$

$\operatorname{Idfm}=\operatorname{decoding}(\operatorname{Imf})$

$\operatorname{Imf}=\operatorname{IDWT}(\mathrm{Idfm})$

This pseudo code shows the implementation Process.

\subsection{Self-Organizing Migrating Algorithm}

The algorithm, Proposed by prof. Zelinka in 1999. This section presents the most common All-to-One SOMA.

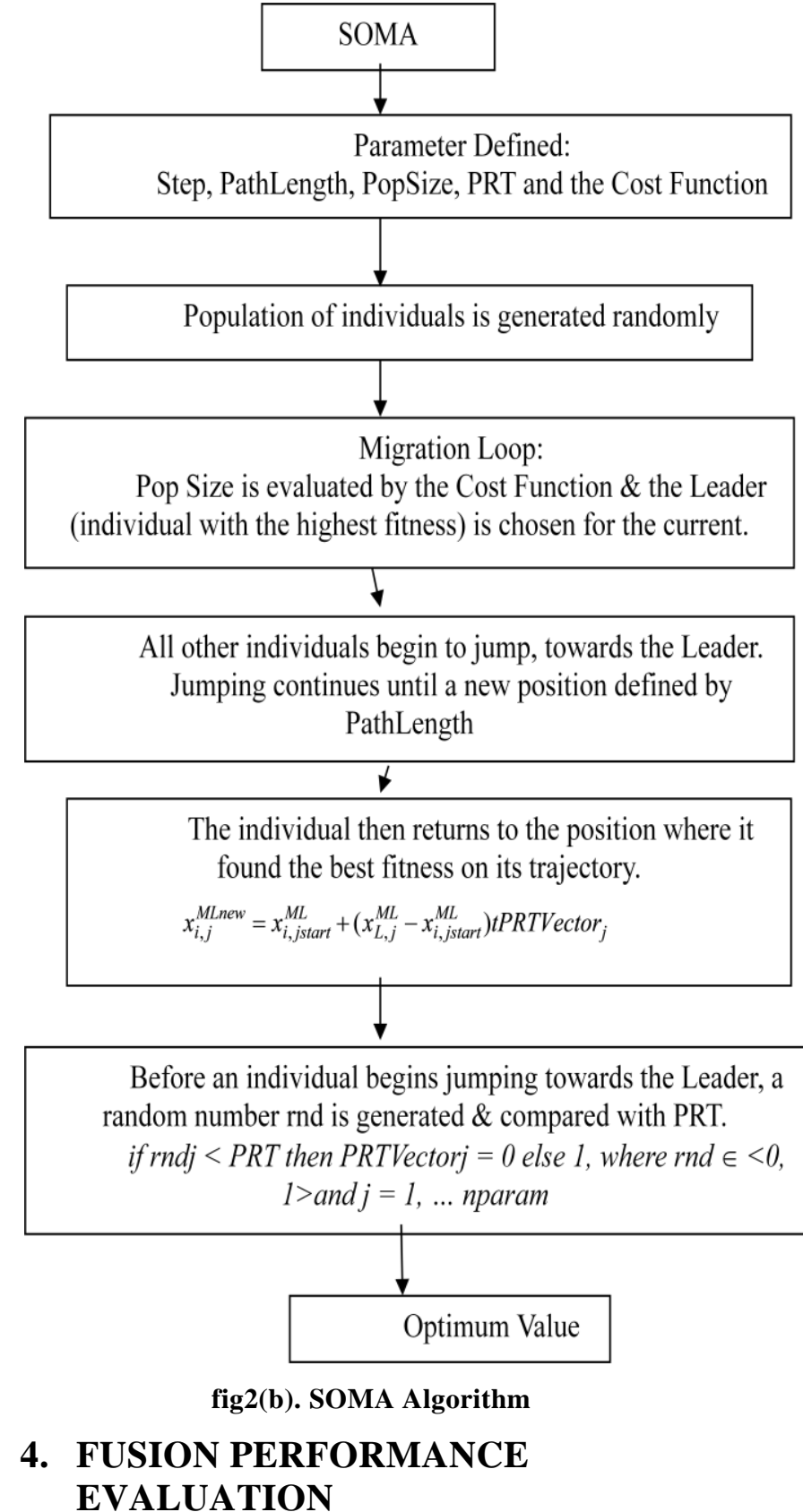

In image fusion algorithms the performance parameters can be evaluated as following parameters $[3,5,8,9]$ :

\subsection{Root Mean Square Error}

Root mean square error (RMSE) is the measure of the corresponding pixels in the true image It and the fused image If. When the true and fused images are identical error generated will be nearly zero and it will enhance when the dissimilarity between images raise.

$$
R M S E=\sqrt{\frac{1}{M N} \sum_{x=1}^{M} \sum_{y=1}^{N}\left(I_{r}(x, y)-I_{f}(x, y)\right)^{2}}
$$

\subsection{Peak Signal to Noise Ratio [PSNR]}

It gives enhanced value when the fused and true images are identical and superior value entails superior fusion. 


$$
P S N R=20 \log _{10}\left(\frac{L^{2}}{\frac{1}{M N} \sum_{x=1}^{M} \sum_{y=1}^{N}\left(I_{r}(x, y)-I_{f}(x, y)\right)^{2}}\right)
$$

Here, L indicates the number of gray levels.

\section{RESULTS AND COMPARATIVELY STUDY}

True image img1.jpg of size $512 \times 512$ is revealed in fig- 3 . The 2 images to be fused are produced from the true image as revealed in fig - $4 \& 5$. The fused image is nearly analogous to true image and the error image is approximately zero. Table- 1 reveals the fusion quality evaluation metrics. The table shows proposed hybrid technique where metrics demonstrated in dark font are superior amongst others. We got almost similar performance by using fusion with 3 level pyramids or some higher values . By applying projected algorithm results are improved as error values are reduced which enhances PSNR values.

\section{CONCLUSION}

An innovative image fusion technique using SPIHT and SOMA has been proposed and its performance is evaluated. We realized better fusion quality by enhancing the fusion levels till 3 . This method can be efficiently used for fusion of images with multi spectral images also. The projected algorithm is easier and efficient to implement for real time applications. This paper also provides a comparative study between proposed \& literature techniques and validation of the projected algorithm as in table 1 .

\section{REFERENCES}

[1] M. Kumar et.al., "Digital Image Watermarking using Fractional Fourier transform via image compression", In IEEE International Conference on Computational Intelligence and Computing Research 2013 (IEEE ICCIC-2013), 26-28 Dec., 2013.

[2] VPS Naidu, "A Novel Image Fusion Technique using DCT based Laplacian Pyramid", International Journal of Inventive Engineering and Sciences (IJIES) ISSN: 23199598, Volume-1, Issue-2, January, 2013.

[3] Mahendra Kumar et. Al..’'Fuzzy type Image Fusion using SPIHT Image compression technique", International Journal of Engineering Research and Applications (IJERA), (Accepted). (Impact factor: 1.69)

[4] Mahendra Kumar et. al.," Digital Image Watermarking using Fractional Fourier Transform with Different Attacks" International Journal of Scientific Engineering and Technology, Volume No.3 Issue No.8, Aug. 2014, pp : 1008-1011. ((ISSN : 2277-1581))

[5] Mahendra Kumar," Face Recognition using SOM Neural Network with Different Facial Feature Extraction Techniques" International Journal of Computer Applications (IJCA), Vol. 76, No. 3, Aug. 2013, pp.7-11. (Impact factor: 0.821)

[6] S. Prabha, Dr. M. Sasikala, "Texture Classification Using Curvelet Transform", International Journal of Advancements in Research \& Technology, Volume 2, Issue4, April-2013.

[7] Nisha Soni et. al.," A Matlab Based High Speed Face Recognition System Using SOM Neural Networks" International Journal of Engineering Research and Applications (IJERA), Vol. 3, Issue 4,July-Aug. 2013, pp.785-790. (Impact factor: 1.69)

[8] Rajesh Kumar Kakerda et. Al.,"Fuzzy type Image Fusion using hybrid DCT-FFT based Laplacian Pyramid Transform", $4^{\text {th }}$ IEEE International Conference on Communication and Signal Processing (ICCSP 2015) 0204 April 2015 - Melmaruvathur, TN, IND.

[9] M. Kumar et. Al.," Comparative Study Of Different Classifiers Based Speaker Recognition System Using Modified MFCC For Noisy Environment", International Conference Green Computing and Internet of Things (ICGCIoT - 2015) 08-10 Oct., 2015, Delhi, IND.

[10] M. Kumar et. A1.,” Robust Digital Image Watermarking using DCT based Pyramid Transform via image compression", $4^{\text {th }}$ IEEE International Conference on Communication and Signal Processing (ICCSP 2015) 0204 April 2015 - Melmaruvathur, TN, IND.

Fig.5: Saras Image 2 
[11] Swati Jadon et. Al.,"Face Recognition Using SOM Neural Network with DDCT Facial Feature Extraction Techniques", $4^{\text {th }}$ IEEE International Conference on Communication and Signal Processing (ICCSP 2015) 0204 April 2015 - Melmaruvathur, TN, IND.

[12] Reema Jain et. Al.," Digital Image Watermarking using Hybrid DWT - FFT Technique with Different Attacks", $4^{\text {th }}$ IEEE International Conference on Communication and Signal Processing (ICCSP 2015) 02-04 April 2015 Melmaruvathur, TN, IND

[13] V.P.S. Naidu, "Novel Image fusion techniques using DCT ", International Journal of Computer Science and Business Informatics, Vol.5, No.1(2013).

[14] Mahendra Kumar et. Al.," Robust Image Fusion based on Optimal Cuve-let Transform", International Conference on Micro-Electronics and

\section{APPENDIX}

\section{Table 1 Comparative Analysis}

*Root Mean Square Error(RMSE);Percentage Fit Error(PFE);Mean Absolute Error(MAE);Correlation(CORR);Signal to Noise Ratio(SNR);Peak Signal to Noise Ratio(PSNR);Mutual Information(MI);Universal Quality Index(UQI);Measure of Structural Similarity Index(SSIM)[8]

*Frequency Partition DCT Image fusion;Laplacian Pyramid DCT Image fusion(LPDCT);1D Multi Resolution DCT (MRDCT)

\begin{tabular}{ccccccccccc}
\hline Technique & RMSE & PFE & MAE & CORR & SNR & PSNR & MI & UQI & SSIM \\
Proposed & $\mathbf{0 . 0 6 4 3}$ & $\mathbf{7 . 2 1 8 0}$ & $\mathbf{0 . 0 4 0 5}$ & $\mathbf{0 . 9 9 7 4}$ & $\mathbf{2 2 . 8 3 1 7}$ & $\mathbf{6 0 . 0 8 1 4}$ & $\mathbf{1 . 5 9 8 8}$ & $\mathbf{0 . 1 2 5 5}$ & $\mathbf{0 . 9 9 9 7}$ \\
\hline FPDCT[13] & 18.2385 & 7.8243 & 16.8633 & 0.9971 & 22.1311 & 35.5549 & 1.1064 & 0.1521 & 0.7888 \\
\hline LPDCT[13] & 3.4201 & 1.4672 & 1.6386 & 0.9999 & 36.6700 & 42.8244 & 1.2884 & 0.3728 & 0.9827 \\
MRDCT[13] & 7.1313 & 3.0593 & 4.9386 & 0.9995 & 30.2875 & 39.6331 & 1.1106 & 0.1568 & 0.8236 \\
\hline
\end{tabular}

